\title{
Zinc-Salt-Mediated Synthesis, Growth Kinetic, and Shaped Evolution of Silver Nanoparticles
}

\author{
E. O. Dare, ${ }^{1,2,3,4}$ O. W. Makinde, ${ }^{2}$ K. T. Ogundele, ${ }^{2}$ G. A. Osinkolu, ${ }^{2}$ Y. A. Fasasi, ${ }^{2}$ I. Sonde, ${ }^{1}$ \\ J. T. Bamgbose, ${ }^{1}$ M. Maaza, ${ }^{3}$ J. Sithole, ${ }^{3}$ F. Ezema, ${ }^{5}$ and O. O. Adewoye ${ }^{4}$ \\ ${ }^{1}$ Department of Chemistry, University of Agriculture, Abeokuta, Nigeria \\ ${ }^{2}$ Center for Energy Research and Development, Obafemi Awolowo University, Ile-Ife, Nigeria \\ ${ }^{3}$ Nanosciences Laboratories, Materials Research Department, iThemba LABS, Somerset West 7129, South Africa \\ ${ }^{4}$ National Agency for Science and Engineering Infrastructure, Idu Industrial Area, Abuja, Nigeria \\ ${ }^{5}$ Department of Physics, University of Nigeria, Nsukka, Nigeria
}

Correspondence should be addressed to E. O. Dare, dare3160@hotmail.com

Received 12 August 2012; Accepted 12 September 2012

Academic Editors: N. R. Jana and S.-H. Kim

Copyright () 2012 E. O. Dare et al. This is an open access article distributed under the Creative Commons Attribution License, which permits unrestricted use, distribution, and reproduction in any medium, provided the original work is properly cited.

\begin{abstract}
We report the synthesis of various shaped silver nanoparticles mediated by $\mathrm{ZnCl}_{2}$ salt. It has been demonstrated that the salt, $\mathrm{PVP} / \mathrm{AgNO}_{3}$ mole ratio, and the type of polyol (EG, DEG, and GL) significantly determined twinning probability, which is an index of silver seed growth origin. High twining probability arising from low PVP/Ag ${ }^{+}$ratio and 50 mole $\%$ salt favours $1 \mathrm{D}$ grown nanowires and nanorods, whereas low twining amidst high mole $\%$ salt (150) in DEG offered 2D grown nanoflakes and nanosheets. Other shaped silver nanoparticles have been found. Accidentally, we arrived at a core-shell heterostructure of Ag-ZnO nanocomposite with Ag core enrichment when mole\% of the salt was made up to 300. Growth kinetic of nanosphere obtained was monitored, and effect of salt mediation was found crucial. Structural evolution of shaped Ag nanoparticles and nanocomposites have been monitored using XRD, SEM, EDX, TEM, and UV/vis.
\end{abstract}

\section{Introduction}

Nanostructured materials have received broad attention due to their distinguished performance in electronics, optics, and photonics [1]. With reduction in size, novel electrical, mechanical, chemical, and optical properties are introduced, which are largely believed to be the result of surface and quantum confinement effects. They have been widely exploited for use in photography, catalysis, biological labeling, photonics, optoelectronics, information storage, and formulation of magnetic ferrofluids [2-4].

Among the various metal nanostructures, silver nanoparticles (SNs) have been widely investigated because they exhibit unprecedented optical, electronic, and chemical properties, depending on their sizes and shapes, thus opening many possibilities for diverse technological applications [57].

Generally, silver nanoparticles have been produced by various methods including chemical reduction of silver ions with or without $[8,9]$ stabilizing agents, thermal decomposition of organic solvents, and electrospining [10]. Using these methods [8-14], silver nanoparticles with spherical, octahedral, tetrahedral, hexagonal, cubic, wire, coaxial cable, triangular prism, disc, and belt shapes have been produced. Apart from metal-silver nanobimetallics [15-19], functionalization or core-shell of silver-metal oxide nanocomposites is limited in the literature. $\mathrm{Ag}-\mathrm{ZnO}$ electrical contact material was only recently produced using mechanochemical synthesis route [20]. Polymer functionalized silver has been reported [21]. Furthermore, the presence of various ions has been shown to influence the shape and size of metallic nanostructures produced via the polyol method. Xia group [22] has shown that the presence of iron (II) or iron (III) in the polyol synthesis facilitates the growth of silver nanowires or cubes, depending on the concentration of iron ions. Copper salts [23] have been used to affect polyol reduction of silver nitrate.

In this present paper, we report the synthesis of various shaped silver nanoparticles and zinc oxide-wrapped silver 


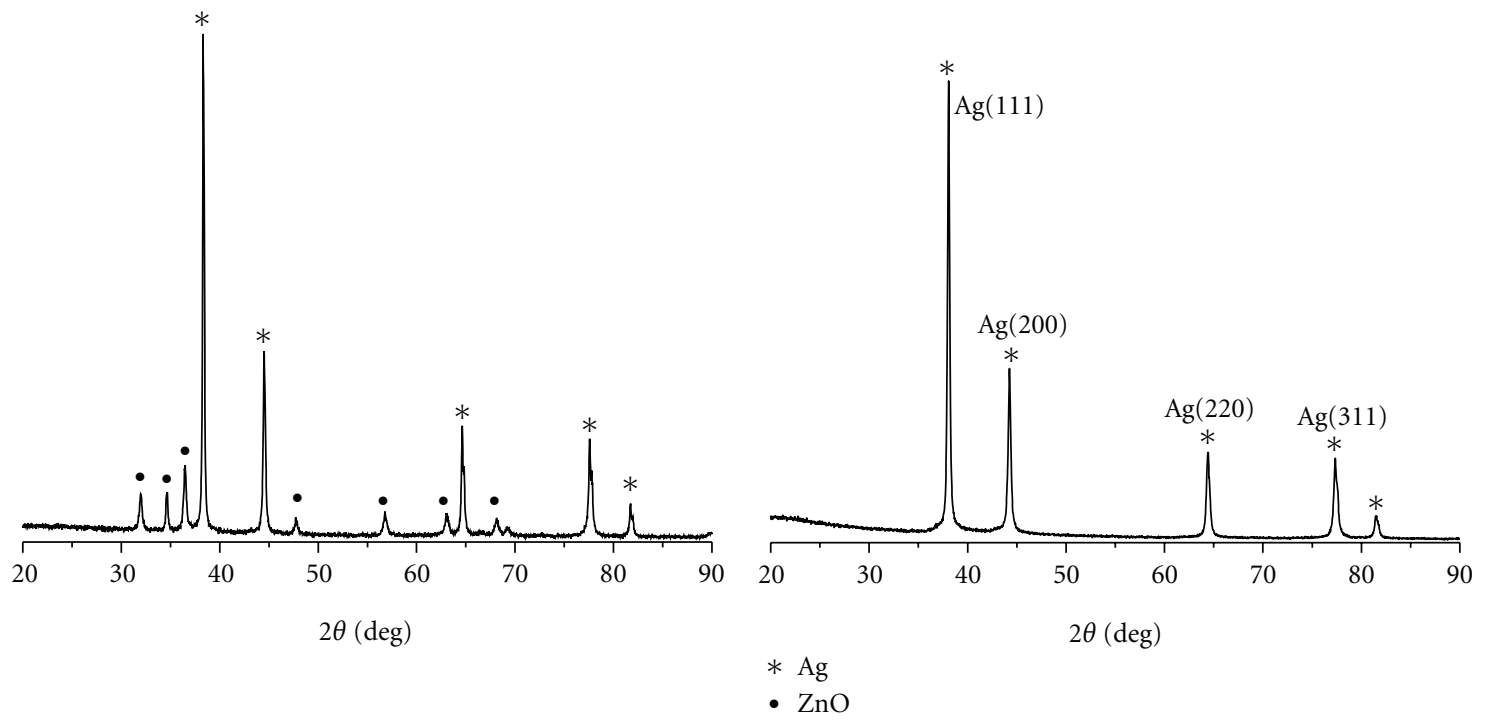

(a)

(b)

FIGURE 1: XRD patterns of as-prepared (a) silver core-ZnO shell nanocomposite, (b) silver sample.

(core-shell) nanocomposite using chemical reduction in a polyol process under the influence of zinc chloride salt. Shapes of the silver and silver core zinc oxide shell nanoparticles have been followed by various techniques such as XRD, TEM, EDX, and UV-vis spectroscopy.

\section{Experimental}

2.1. Materials. The experiments were conducted using analytical grade zinc chloride $\left(\mathrm{ZnCl}_{2}\right)$, Silver nitrate $\left(\mathrm{AgNO}_{3}\right)$, and Polyvinylpyrrolidone (PVP) powder (PVP, lot analysis, $96.2 \%, \mathrm{MW}=1,300000$ ). All solvents (ethylene glycol (EG), diethylene glycol (DEG), and glycerol (GL)) were used without purification. All chemicals and solvents were commercially obtained from Sigma-Aldrich Chemicals.

2.2. Characterization. X-ray diffraction (XRD) measurements were performed on D8 Advance Bruker X-ray diffractometer, with $\mathrm{Cu} K \alpha 1$ radiation $(\lambda=0.154060 \mathrm{~nm}$ at $40 \mathrm{KV}$ and $40 \mathrm{~mA}$ ). Scanning electron microscope (SEM) images were recorded on a field emission scanning electron microscopy (FESEM, Quanta 200F, HV $15.0 \mathrm{kV}$ ) coupled with an energy-dispersive X-ray spectrometer (EDX, Genesis 2000). Transmission electron microscope was carried out using Model 3010 JEOL microscope operating at $300 \mathrm{kV}$. The UV-visible spectra were measured with a Shimadzu UV-2450 spectrometer.

\section{Synthesis}

3.1. Shaped Silver Nanoparticles. The shaped silver nanoparticles were synthesized by chemical reduction method in a polyol process. The polyols used which served as the reducing agents were ethylene glycol (EG), diethylene glycol (DEG), or glycerol (GL). Molar concentrations of reactants were prepared in the probe solvent, unless otherwise stated. PVP served as protective stabilizing agent, while zinc chloride (salt) is expected to facilitate reduction process and seed formation. The molar ratio $\mathrm{PVP} / \mathrm{Ag}^{+}$was set within the range $0.3-2.0 .10 \%-300 \%$ mole of the salt (relative to silver nitrate) was used, unless otherwise stated.

In a typical procedure, $20 \mathrm{~mL}$ of the polyol [EG, DEG, TEG, GL] was heated to $100^{\circ} \mathrm{C}$ with stirring for $30 \mathrm{~min}$. A solution containing $5.0 \mathrm{mM}$ silver nitrate and $0.5 \mathrm{mM}$ (10\%) were added to the stirring polyol (EG, DEG, TEG or GL). Polyol solution of PVP (2.5 mM) was injected over a period of $5 \mathrm{~min}$, and thereafter temperature of $180^{\circ} \mathrm{C}$ was maintained. Reflux condition was used for the coreshell synthesis. The colloidal system was cooled to room temperature, and silver nanoparticles were separated from the liquid by centrifugation and then washed with acetone and ethanol.

\section{Results and Discussion}

Growth and nucleation of SN was apparently noticed as PVP was introduced. The reactant mixture changes quickly from colorless to yellow after the addition of PVP and $\mathrm{ZnCl}_{2}$ salt solution in EG. Yellow to light greenish-opaque colloidal color transition, which was maintained for more than 40 minutes, served to indicate a complete reaction. Turbid brown, wispy gray, and wispy gray colors were the final colors marking a complete reaction in the case when reducing solvents were GL, DEG, and TEG, respectively. The color changes in nanomaterial synthesis are due to confinement of electrons and consequent changes in electronic energy levels [9].

The XRD patterns of a typical silver nanoparticle synthesized in this study are almost identical regardless of experimental conditions (Figure 1(b)), which is a reflection 
TABLE 1: Synthesis conditions for various shaped silver nanoparticles.

\begin{tabular}{|c|c|c|c|c|c|c|c|}
\hline Sample & $\begin{array}{c}\mathrm{PVP}: \mathrm{AgNO}_{3} \\
\text { (mole ratio) }\end{array}$ & $\begin{array}{c}\mathrm{ZnCl}_{2} \\
(\text { mole \%) }\end{array}$ & $\begin{array}{c}\text { Temperature } \\
\left({ }^{\circ} \mathrm{C}\right)\end{array}$ & $\begin{array}{l}\text { Time } \\
(\min )\end{array}$ & Mean size & Polyol & Shape and figure ID (2a-1) \\
\hline 1 & 0.5 & 10 & 180 & 75 & $48 \mathrm{~nm}$ & EG & Nanosphere (c) \\
\hline 2 & 1.0 & 50 & 180 & 75 & $\begin{array}{c}5-30 \mu \mathrm{m} \text { (length) } \\
20-26 \mathrm{~nm} \text { (diameter) }\end{array}$ & EG & Nanowire (h) \\
\hline 3 & 0.3 & 50 & 180 & 75 & $\begin{array}{l}\text { 20-50 } \mu \mathrm{m} \text { (length) } \\
33-42 \mathrm{~nm} \text { (diameter) }\end{array}$ & EG & Nanowire (i) \\
\hline 4 & 0.5 & 30 & 180 & 90 & Not determined & DEG & Nanowire and nanoparticles (g) \\
\hline 5 & 1.0 & 150 & 180 & 90 & 82-131 nm (thickness) & DEG & Nanoflakes (e) \\
\hline 6 & 2.0 & 150 & 180 & 90 & 60-110 nm (thickness) & DEG & Nanosheet (f) \\
\hline 7 & 1.0 & 100 & 180 & 180 & Not determined & GL & Fluffy nanocake (k) \\
\hline 8 & 0.5 & 0 & 180 & 120 & $75-81 \mathrm{~nm}$ & EG & Quazi nanosphere (d) \\
\hline 9 & 1.0 & 0 & 180 & 120 & $41 \mathrm{~nm}$ & EG & Multiply twinned ( $\mathrm{a}$ and $\mathrm{b}$ ) \\
\hline 10 & 2.0 & 0 & 180 & 120 & $\begin{array}{l}\text { 78-83 nm (diameter) } \\
\text { 354-401 nm (length) }\end{array}$ & EG & Nanorod (j) \\
\hline 11 & 1.0 & 300 & reflux & 90 & $110 \mathrm{~nm}$ & EG & $\begin{array}{c}\text { Agglomerated } \\
\text { Ag/ZnO nanocomposite (l) }\end{array}$ \\
\hline
\end{tabular}

of high degree of crystallinity. This indicates that all of the diffraction peaks can be readily indexed to face-centered cubic (fcc) silver with a calculated lattice constant of $4.1 \AA$.

4.1. Effects of Polyols, PVP/Silver Nitrate Mole Ratio, and Salt. It has been established that morphology, sizes, and size distribution of metallic nanoparticles depend on some reaction conditions such as temperature, time, concentration, $\mathrm{PVP} / \mathrm{Ag}^{+}$mole ratio and style of reagents addition [8] as it affects whether nucleation is homogeneous or heterogeneous [24]. The synthesis conditions used for producing the various shaped SNs are summarized in Table 1.

Apparently, nature of the polyol, PVP/silver nitrate ratio, and fraction of the salt added affected shapes, sizes, and reaction time significantly. The reaction in the presence of EG where PVP/Ag mole ratio (0.5) and 50 mole\% $\mathrm{ZnCl}_{2}$ salt were adopted produced nanowires whose length and diameter are in the range $5-30 \mu \mathrm{m}$ and $20-26 \mathrm{~nm}$, respectively (Table 1, Figure $2(\mathrm{~h})$ ).

Obviously, Ag nanowires must have been generated following self-seeding process previously explained $[25,26]$. Relatively lower $\mathrm{Ag}^{+}$precursor concentration accounts for a reduced chemical potential which favours thermodynamically stable multiply-twinned particles (MTPs) (Table 1, Figures 2(a), and 2(b)). Maintaining that condition with $\mathrm{Cl}^{-}$addition favours oxidative etching of the MTPs and the resultant overriding growth led to nanowires (Figure 2(h)). However, there was a significant change in length and diameter of the nanowires as the $\mathrm{PVP} / \mathrm{Ag}^{+}$is reduced to 0.3 while keeping $50 \%$ salt addition. This led to increased growth rate of the etched MTPs, leading to longer and thicker nanowires (Figure 2(i)). A report elsewhere [22] indicated contributory effort of $\mathrm{Fe}^{2+}$ in promoting production of nanowires. $\mathrm{Cu}^{2+}$ has also aided reduction of $\mathrm{AgNO}_{3}$ [23]. At $\mathrm{PVP} / \mathrm{Ag}^{+}$ratio and $10 \%$ Zinc salt intervention, a perfect nanosphere (Table 1, Figure 2(c)) of diameter $48 \mathrm{~nm}$ resulted within $75 \mathrm{~min}$. However, a cross-check experiment under the same condition and without salt mediation offered quazi nanosphere in 3 hours reaction time.

Scheme 1 explained the formation of shaped Ag which, however, depends on the level of $\mathrm{Cl}^{-}$mediation vis-à-vis the nature of polyol. Following path 8 and 9 of the scheme, $\mathrm{Ag}^{+}$ is reduced to $\mathrm{Ag}^{\mathrm{o}}$, nucleation occurs, and seed formation leads to quazi nanosphere whose seed growth proceed in an isotropic manner (OD particles). Since nanosphere belongs to the lowest energy shape, simple reduction of the silver precursor is expected with or without exotic reagent. In this case, synergy effect of the salt has been noted towards formation of a perfect nanosphere within $75 \mathrm{~min}$ in hopefully kinetically controlled manner. Hopefully, the synergy efforts of both $\mathrm{Zn}^{2+}$ and PVP lowered $\{100\}$ surface energy and brought down reaction time to $75 \mathrm{~min}$ from 120 min spent without salt contribution.

PVP has been shown to preferentially adsorb onto the $\{100\}$ surface of silver particles, where it served to stabilize and protect the small single crystal seeds [27]. Insufficient molar concentration of PVP to lower surface energy usually give room for the production of twin detects via a higher surface energy [24].

The morphology of the seed particles is one important parameter that decides anisotropic growth of noble metal nanoparticles; the process that supports the type of shape obtained is dependent on twinning probability [28]. Following reaction Scheme 1 shaped anisotropic growth, $\mathrm{Ag}$ emanated from both $\mathrm{AgCl}$ and $\mathrm{Ag}^{+}$(path 2 and 3) due to large excess of $\mathrm{AgNO}_{3}$ present in the solution. The only few $\mathrm{Cl}^{-}$came from $\mathrm{AgCl}$, which dissolved as the temperature is increased beyond $150^{\circ} \mathrm{C} . \mathrm{Ag}^{+}$concentration in the medium is sufficient enough to promote twinning process amidst some oxidative etching effect of $\mathrm{Cl}^{-} / \mathrm{O}_{2}$ of the initially formed MTPs. Under this condition, the growth rate from decahedra to $1 \mathrm{D}$ anisotropic products is faster and it competes overwhelmingly with etching rate. In DEG, high $\mathrm{Ag}^{+}$concentration around the growing nuclei pushes in 


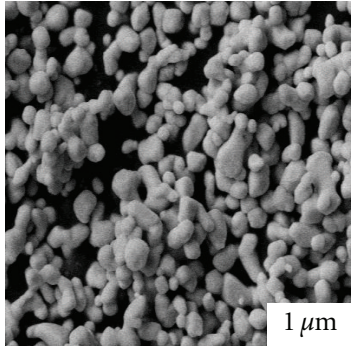

(a)

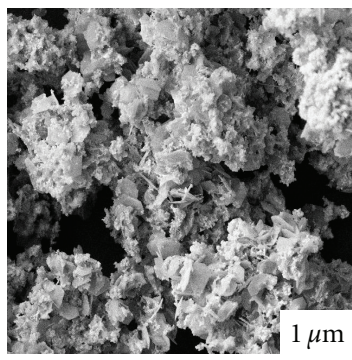

(e)

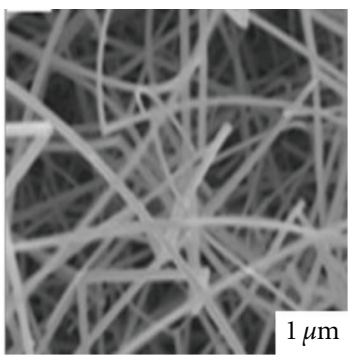

(i)

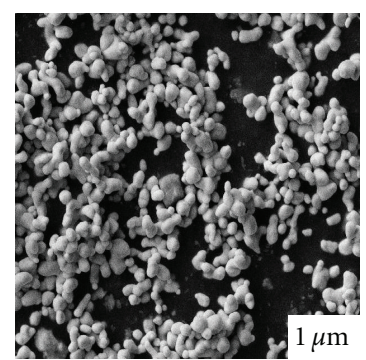

(b)

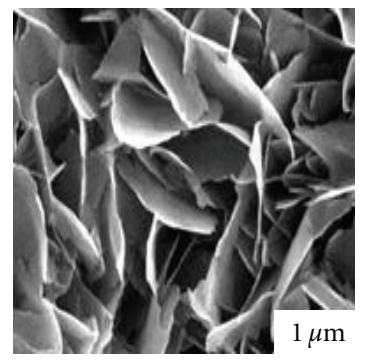

(f)

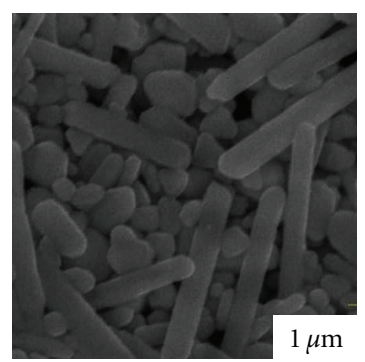

(j)

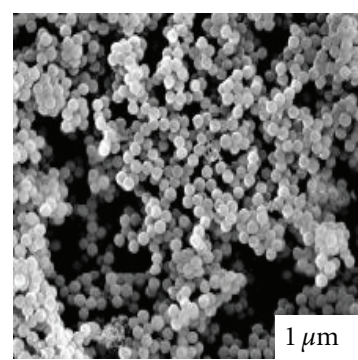

(c)

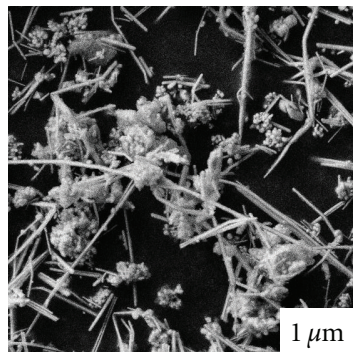

(g)

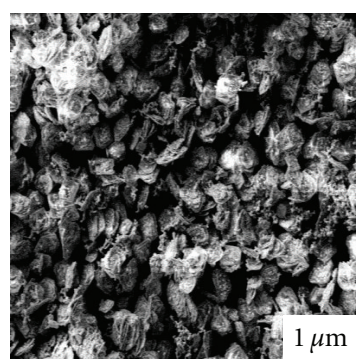

(k)

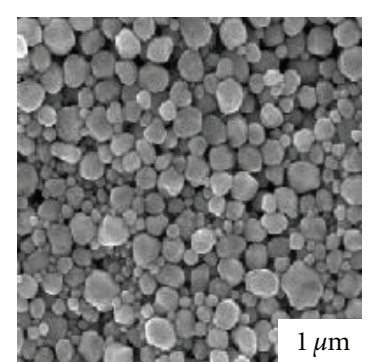

(d)

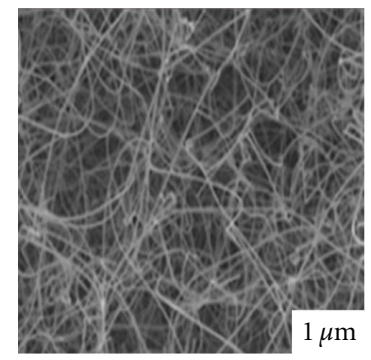

(h)

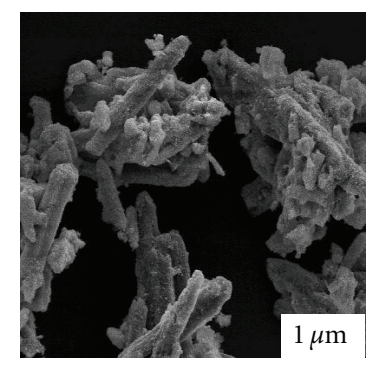

(1)

FIGURE 2: (a-1) Representative SEM images of various shaped Ag synthesis under salt-mediated polyol process.

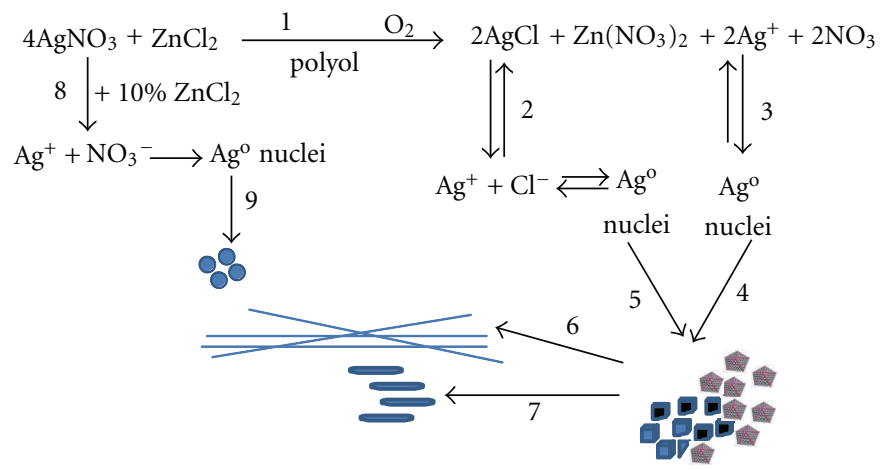

Scheme 1: Proposed synthetic pathway for some shaped Ag nanoparticles when low mole\% of the salt was used.

high twining probability requisite for MTPs formation from where nanorod and nanowires were grown.

As the concentration of $\mathrm{Ag}^{+}$is reduced and $\mathrm{Cl}^{-}$in the reaction medium significantly increased while keeping DEG as reducing solvent (Scheme 2), the morphologies of the Ag formed changed beyond scientific expectation and tuned towards 2D anisotropic nanostructures (nanoflakes and nanosheets) depending on the amount of $\mathrm{Cl}^{-}$in the reaction medium as indicated in the table. The plausible explanation to this observation is that $\mathrm{Ag}^{\mathrm{O}}$ atoms around the growing Ag nuclei are significantly low and that prevented twinning probability for MTPs. Therefore, The scenario displayed promoted extensively high $\mathrm{Cl}^{-} / \mathrm{O}_{2}$ oxidative etching which may lead to unequally grown $\{100\}$ and $\{111\}$ facets of silver. 


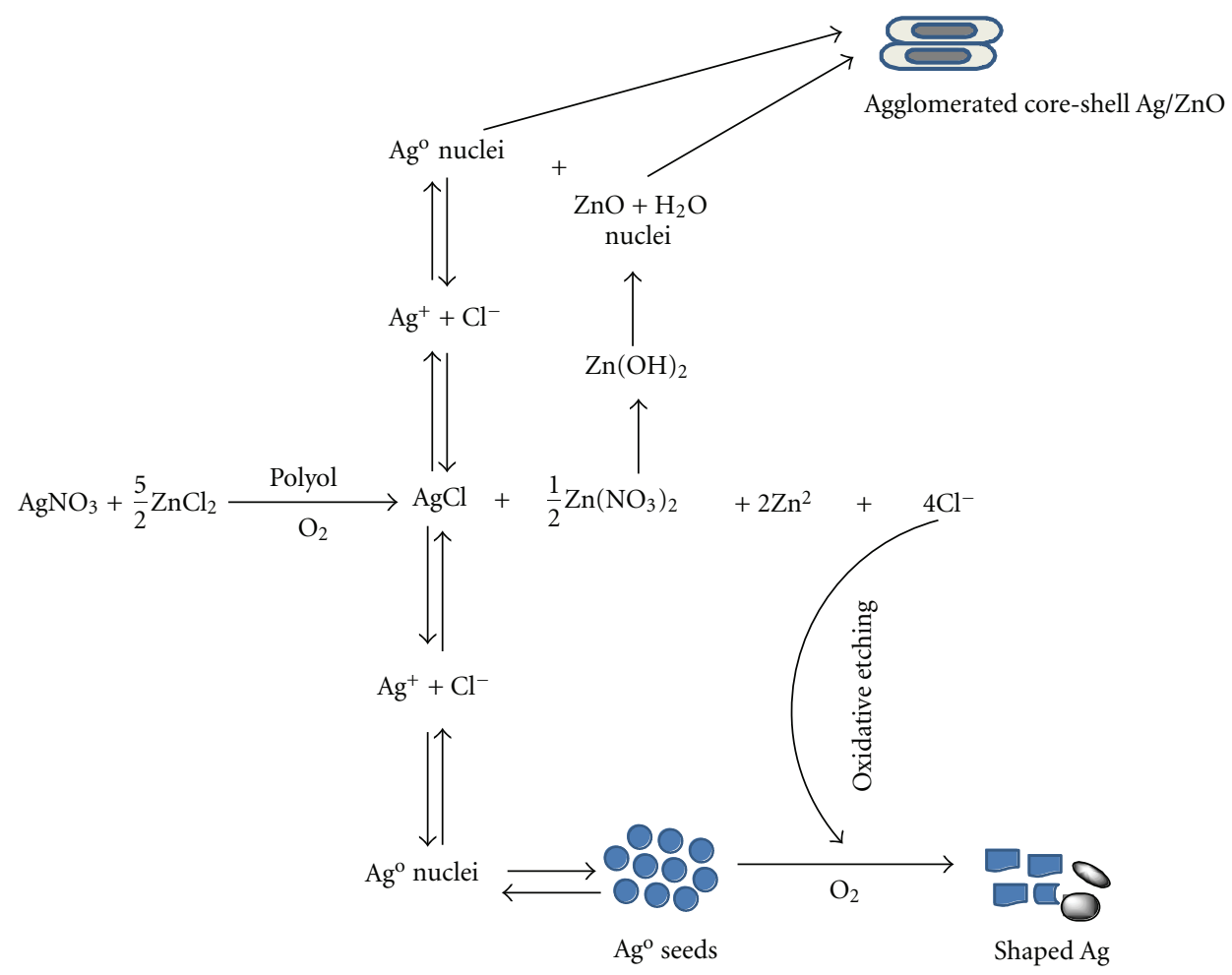

Scheme 2: Proposed synthetic pathway for some shaped Ag nanoparticles when high mole\% of the salt was used.

Eventually, $\mathrm{Cl}^{-}$supposedly suppresses the crystal growth along the $\{111\}$ direction [29]. Obviously, the area ratio of $\{100\} /\{111\}$ facet varies depending on the relative growth rate of the two surfaces, resulting in differences in particle shapes [29]. Therefore, the formation and structural changes observed for nanoflakes $\rightarrow$ nanosheets can be explained by the $\mathrm{Cl}^{-} / \mathrm{O}_{2}$ etching vis-à-vis site-specific deposition of $\mathrm{AgCl}$ on the surface of Ag nanoparticle seeds [30]. In other words, selective adsorption of $\mathrm{Cl}^{-}$on the $\{111\}$ plane of $\mathrm{Ag}$ seeds and the formation of $\mathrm{AgCl}$, also in contact with the seeds, play a role in the nanosheets and nanoflakes formation as promoted by DEG.

Interestingly, reaction in the presence of $\mathrm{GL}$ while keeping equal ratio of $\mathrm{PVP} / \mathrm{Ag}^{+}$offered what we may name as a fluffy round nanocake (Figure $2(\mathrm{k})$ ). This observation further shows that the type of aldehyde emanated from the polyol, which in this case GL, causing the reduction process is also important in the shape determination of the growing silver seeds. The predominating anisotropic $1 \mathrm{D}$ nanorod (78 $\mathrm{nm}$ diameter, $352 \mathrm{~nm}$ length on the average, Figure 2(j)) obtained is similar to that previously reported [31].

To further clarify, if indeed $\mathrm{Zn}^{2+} \mathrm{Cl}^{-}$has a significant effect on the morphological changes of the nanoparticles, one may ask then what happens when $\mathrm{Cl}^{-}$is greatly increased. To answer this, mole $\%$ of $\mathrm{ZnCl}_{2}$ was made up to 300 and the reaction was carried out under reflux condition. Representative SEM spectrum in Figure 2(l) shows what we may call $\mathrm{ZnO}$-wrapped Ag nanocomposite, which obviously followed a core-shell model as monitored by UV-vis absorption spectroscopy (spectra not shown for brevity) and XRD.
The XRD pattern (Figure 1(a)) of the as-prepared Ag core- $\mathrm{ZnO}$ shell is quite revealing and distinct in terms of crystallinity and peaks attributable to the heterostructure (OD on 1D). This exceptional distinct nature of the crystalline phases peaks rules out the possibility for an alloy formation as there is no evidence of intimate association of $\mathrm{ZnO}$ within $\mathrm{Ag}$ matrix. The $\mathrm{C}_{\mathrm{Ag}} / \mathrm{C}_{\mathrm{ZnO}}$ ratio in the coreshell is ca 6.4, indicating a large core enrichment of the Ag. Structural evolution of Ag-ZnO (Figure 1(b)) from Ag (Figure 1(a)) has been demonstrated with the emanation of peaks (1010), (0002), (1011), (1012), (1120), and (1013) which are due to $\mathrm{ZnO}$ nanostructure.

The plausible mechanism of formation of agglomerated $\mathrm{Ag}-\mathrm{ZnO}$ can be followed from Scheme 2. Obviously, Ag nanorod resulted from dissolving $\mathrm{AgCl}$ and $\mathrm{Zn}\left(\mathrm{NO}_{3}\right)_{2}$ offererd $\mathrm{Zn}(\mathrm{OH})_{2}$ precipitate, which was transformed into $\mathrm{ZnO}$ nuclei. The $\mathrm{ZnO}$ nuclei grew and self-assembled itself on the Ag nanorod surface due to relative surface energy concepts.

The morphologies and structures of some of the shaped silver nanoparticles were investigated by TEM, EDX, and UVvis spectroscopy. Figures $3(\mathrm{a})$ and $3(\mathrm{~b})$ are the representative TEMs of the anisotropic 1D structures of nanorod and nanowire, respectively, which are crystalline in nature. Electron diffraction pattern recorded from Figure 3(b) displays characteristic rings which could be index as the (111), (200), (220), and (311) allowed reflections from fcc silver.

Growth kinetic of the nanosphere seed formation was studied with the view to further clarify the influence of $\mathrm{Zn}^{2+}$ $\mathrm{Cl}^{-}$as it affects reaction rate. The absorption of silver at 


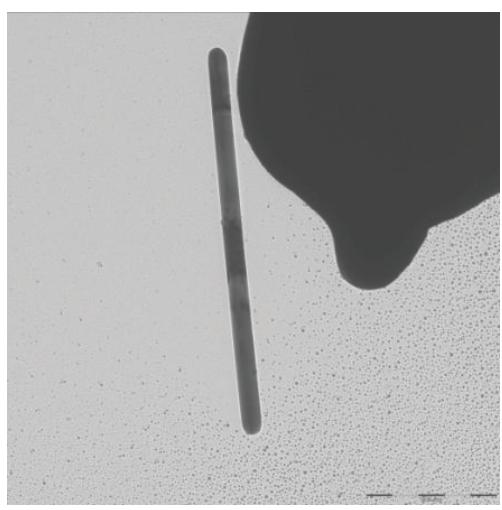

(a)

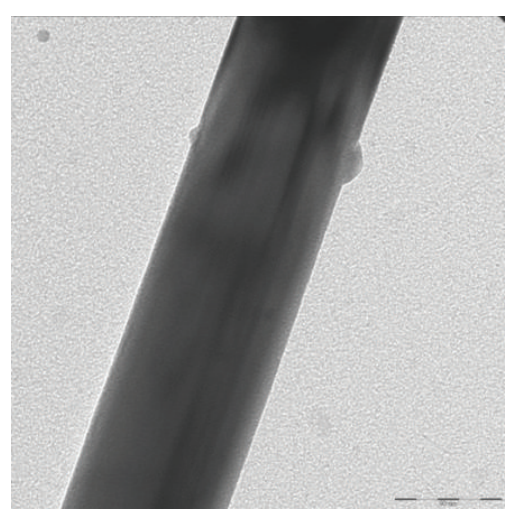

(b)

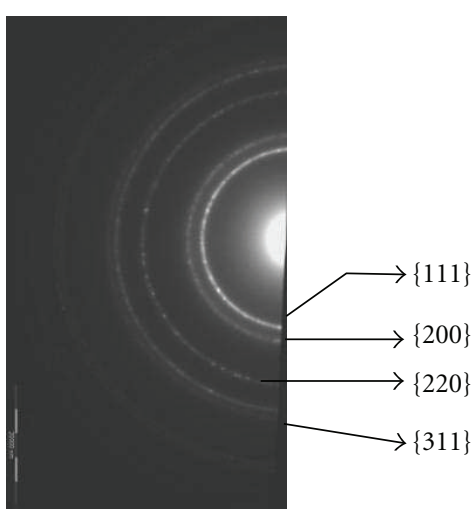

(c)

FIgURE 3: Representative TEM images of (a) Ag nanorod, (b) Ag nanowire obtained at different levels of salt mediation, (c) electron diffraction pattern recorded from the Ag nanowire shown in image (b).

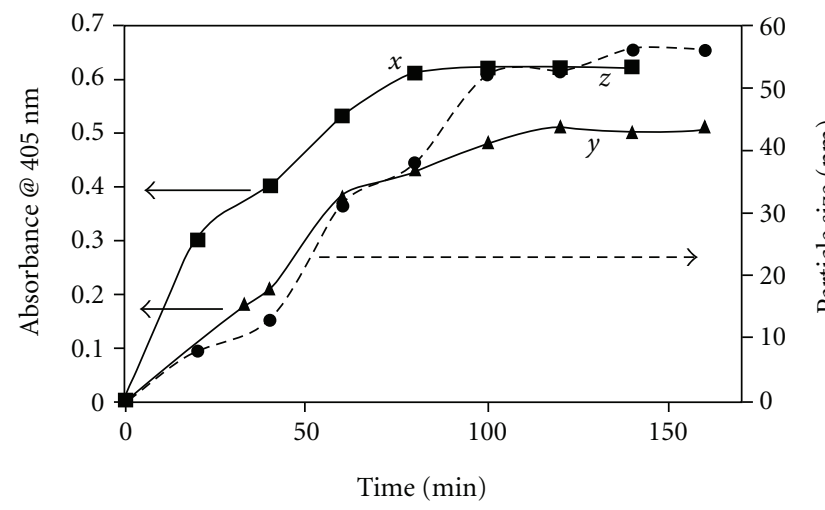

(a)

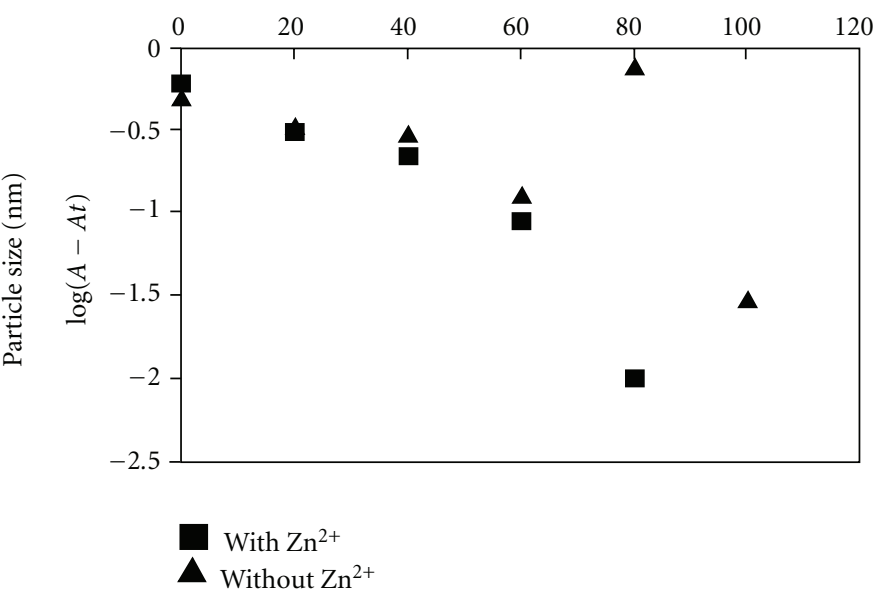

(b)

FIGURE 4: Growth kinetic of the nanosphere formation with or without salt mediation (a) plots of absorbance and particle sizes against time, (b) plot of $\log (A-A t)$ against time.

$405 \mathrm{~nm}$ and corresponding particle sizes are plotted against time (Figure 4(a)). Curve $z$ (dashed line - - ) represents the particle sizes evolution as the nanosphere grew. Curve $x$ and curve $y$ are those with and without $\mathrm{ZnCl}_{2}$ salt, respectively. In both cases, absorbance and particle size increased with increasing time until when equilibrium was attained after which there was no further growth. However, absorbance of Ag nanosphere under the mediating influence of salt was significantly higher (curve $x$ ), indicating increased particle density. A plot of $\log (A-A t)$ against time (Figure 4(b)) is quite revealing and concluded the effect of salt mediation. Calculated rate constant $\left(k=4.6 \times 10^{-1} \mathrm{~S}^{-1}\right)$ under salt influence has been found to be almost three times higher than that without salt mediation $\left(k=1.6 \times 10^{-2} \mathrm{~S}^{-1}\right)$. Apparently, nucleation and growth process vis-à-vis particle size of the perfect nanosphere could be kinetically controlled to reach equilibrium and which eventually brought the reaction to completion within $75 \mathrm{~min}$ in contrast to that which was not mediated, taking up to $120 \mathrm{~min}$ reaction time. Both cation and anion may be responsible for this observation [32]. Certainly, $\mathrm{Zn}^{2+}$ influenced the rate of reaction and size equilibrium, while $\mathrm{Cl}^{-}$is responsible for the shape of nanoparticles formation.

It has been established that copper, gold, and silver display plasmon absorption in the visible region. However, silver in particular usually has an absorption maximum at $404 \mathrm{~nm}$ [33]. The UV-visible spectra of silver nanosphere, nanorod, and nanowires synthesized are showed in Figure 5 with corresponding curves $x, y$, and $z$, respectively. $\mathrm{Ag}$ nanosphere, which is of the lowest energy, displays absorption maximum at $421 \mathrm{~nm}$, while $\mathrm{Ag}$ nanorod and $\mathrm{Ag}$ nanowire show red-shifted plasma absorption maximum at $452 \mathrm{~nm}$ and $484 \mathrm{~nm}$, respectively. The reason behind the discrepancies in the observed optical properties is attributed to increase in aspect ratio as the morphologies change from spheres to rods and wires; the larger the aspect ratio, the more red-shifted the longitudinal Plasmon band [34, 35]. Moreover, the shifting experienced by the nanorod and 


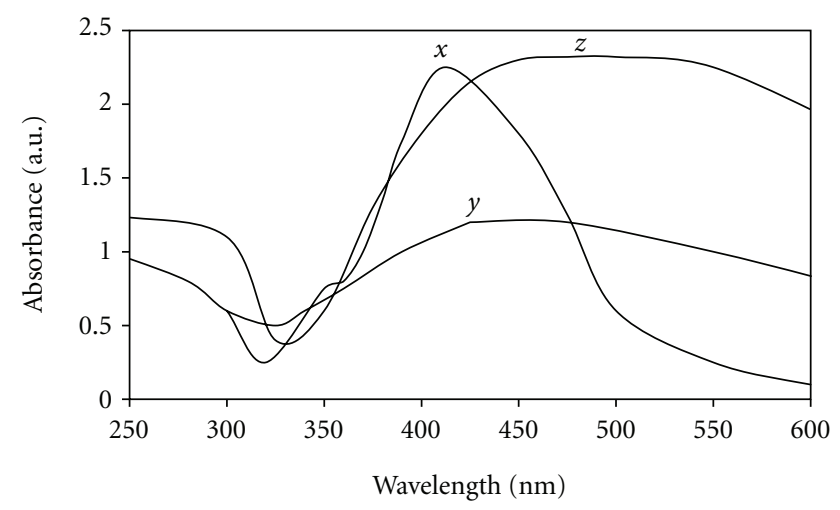

Figure 5: UV-vis Absorption spectrum of $(x)$ nanosphere $(y)$, nanorod, and $(z)$ nanowire.

nanowire is due to enhanced absorption of visible light along their respective length and width [35]. Nanowire is longer and is therefore more red-shifted.

It is worth noting that accidentally acquired results obtained on the core-shell $\mathrm{Ag} / \mathrm{ZnO}$ heterostructure, though agglomerated, seems hopeful if further experimental measures are taken. Study in this direction on full scale basis is underway.

In conclusion, it has been established that $\mathrm{ZnCl}_{2}$ salt vis-à-vis variations in $\mathrm{PVP} / \mathrm{AgNO}_{3}$ ratio and type of polyol, to a large extent, have influence on the nucleation, growth, and final shapes of silver nanostructure. The mole $\%$ of the salt relative to the silver precursor determined whether the $\mathrm{Ag}$ seed formation proceeded in an isotropic or anisotropic manner, which in this case was found to depend on twinning probability and amount of $\mathrm{Cl}^{-}$and $\mathrm{Ag}^{+}$in the reaction mixture. Ag shape formation was either initiated via seed growth arising from MTPs leading to nanowires and nanorods or via $\mathrm{Cl}^{-} / \mathrm{O}_{2}$ leading to other shapes (flakes, sheets, cake, etc.) depending on the extent of etching and polyol used. Extremely high mole $\%$ of the salt has been found to be contributory factor to the development of Ag core$\mathrm{ZnO}$ shell heterostructure bearing OD on 1D. In particular, the strategies to control the kinetic and formation of desired nuclei with twin planes during nucleation offered option for anisotropically shaped silver nanostructures. The type of polyols adopted has been found to be responsible for that option.

\section{Acknowledgment}

The authors thank Centre for Energy Research and Development (CERD), Obafemi Awolowo University, IleIfe, Nigeria, and Nanotechnology for Africa Network (NANOAFNET), ithemba Labs, Somerset West, South Africa, and National Agency for Science and Engineering Infrastructure (NASENI), Abuja, Nigeria, for financial contribution. E. O. Dare was at these places as a Visiting Scientist. They also appreciate Professor P. Kalu of Mechanical Engineering division, Florida state University, USA, for SEM and TEM measurements of some of the samples.

\section{References}

[1] C. M. Lieber, "Nanoscale science and technology: building a big future from small things," MRS Bulletin, vol. 28, no. 7, pp. 486-491, 2003.

[2] M. P. Pileni, "Magnetic fluids: fabrication, magnetic properties, and organization of nanocrystals," Advanced Functional Materials, vol. 11, no. 5, pp. 323-336, 2001.

[3] S. Nie and S. R. Emory, "Probing single molecules and single nanoparticles by surface-enhanced Raman scattering," Science, vol. 275, no. 5303, pp. 1102-1106, 1997.

[4] P. V. Kamat, "Photophysical, photochemical and photocatalytic aspects of metal nanoparticles," The Journal of Physical Chemistry B, vol. 106, no. 32, pp. 7729-7744, 2002.

[5] Y. Sun, Y. Yin, B. T. Mayers, T. Herricks, and Y. Xia, "Uniform silver nanowires synthesis by reducing $\mathrm{AgNO}_{3}$ with ethylene glycol in the presence of seeds and poly(vinyl pyrrolidone)," Chemistry of Materials, vol. 14, no. 11, pp. 4736-4745, 2002.

[6] D. Zhang, J. Yang, J. Ma, H. Cheng, and L. Huang, "Wet chemical synthesis of silver nanowire thin films at ambient temperature," Chemistry of Materials, vol. 16, no. 5, pp. 872876, 2004

[7] L. Suber, I. Sondi, E. Matijević, and D. V. Goia, "Preparation and the mechanisms of formation of silver particles of different morphologies in homogeneous solutions," Journal of Colloid and Interface Science, vol. 288, no. 2, pp. 489-495, 2005.

[8] D. Kim, S. Jeong, and J. Moon, "Synthesis of silver nanoparticles using the polyol process and the influence of precursor injection," Nanotechnology, vol. 17, no. 16, pp. 4019-4024, 2006.

[9] L. M. Liz-Marzan and A. P. Philipse, "Stable hydrosols of metallic and bimetallic nanoparticles immobilized on imogolite fibers," The Journal of Physical Chemistry, vol. 99, no. 41, pp. 15120-15128, 1995.

[10] M. Jin, X. Zhang, S. Nishimoto et al., "Large-scale fabrication of Ag nanoparticles in PVP nanofibres and net-like silver nanofibre films by electrospinning," Nanotechnology, vol. 18, no. 7, Article ID 075605, 2007.

[11] A. R. Siekkinen, J. M. McLellan, J. Chen, and Y. Xia, "Rapid synthesis of small silver nanocubes by mediating polyol reduction with a trace amount of sodium sulfide or sodium hydrosulfide," Chemical Physics Letters, vol. 432, no. 4-6, pp. 491-496, 2006.

[12] J. Zhu, C. Kan, X. Zhu et al., "Synthesis of perfect silver nanocubes by a simple polyol process," Journal of Materials Research, vol. 22, no. 6, pp. 1479-1485, 2007.

[13] S. Chen and D. L. Carroll, "Synthesis and characterization of truncated triangular silver nanoplates," Nano Letters, vol. 2, no. 9, pp. 1003-1007, 2002.

[14] A. Callegari, D. Tonti, and M. Chergui, "Photochemically grown silver nanoparticles with wavelength-controlled size and shape," Nano Letters, vol. 3, no. 11, pp. 1565-1568, 2003.

[15] S. H. Liou, S. Huang, E. Klimek, R. D. Kirby, and Y. D. Yao, "Enhancement of coercivity in nanometer-size CoPt crystallites," Journal of Applied Physics, vol. 85, no. 8, pp. 43344336, 1999.

[16] I. Srnova-Sloufova, F. Lednicky, A. Gemperle, and J. Gemperlova, "Core-shell (Ag)Au bimetallic nanoparticles: analysis of transmission electron microscopy images," Langmuir, vol. 16, no. 25, pp. 9928-9935, 2000.

[17] M. P. Mallin and C. J. Murphy, "Solution-phase synthesis of sub-10 nm Au-Ag alloy nanoparticles," Nano Letters, vol. 2, no. 11, pp. 1235-1237, 2002. 
[18] N. Sandhyarani and T. Pradeep, "Crystalline solids of alloy clusters," Chemistry of Materials, vol. 12, no. 6, pp. 1755-1761, 2000.

[19] D.-H. Chen and C.-J. Chen, "Formation and characterization of Au-Ag bimetallic nanoparticles in water-in-oil microemulsions," Journal of Materials Chemistry, vol. 12, pp. 1557-1562, 2002.

[20] P. B. Joshi, V. J. Rao, B. R. Rehani, and A. Pratap, "Silverzinc oxide electrical contact materials by mechanochemical synthesis route," Indian Journal of Pure and Applied Physics, vol. 45, no. 1, pp. 9-15, 2007.

[21] M. S. Park, T.-H. Lim, T. -M Jeon, J.-G. Kim, and M. S. Gong, "Preparation of new polyelectrolyte/silver nanocomposites and their humidity-sensitive properties," Macromolecular Research, vol. 16, no. 4, pp. 308-313, 2008.

[22] B. Wiley, Y. Sun, and Y. Xia, "Polyol synthesis of silver nanostructures: control of product morphology with $\mathrm{Fe}(\mathrm{II})$ or Fe(III) Species," Langmuir, vol. 21, no. 18, pp. 8077-8080, 2005.

[23] Y. Xia, "One-dimensional nanostructures: synthesis, characterization, and applications," Advanced Materials, vol. 15, no. 5, pp. 353-389, 2003.

[24] C. Ducamp-Sanguesa, R. Herrera-Urbina, and M. Figlarz, "Synthesis and characterization of fine and monodisperse silver particles of uniform shape," Journal of Solid State Chemistry, vol. 100, no. 2, pp. 272-280, 1992.

[25] J. Mullin, Crystallization, Oxford University Press, New York, NY, USA, 1997.

[26] L. D. Marks, "Experimental studies of small particle structures," Reports on Progress in Physics, vol. 57, no. 6, pp. 603649, 1994.

[27] Y. Sun, B. Mayers, T. Herricks, and Y. Xia, "Polyol synthesis of uniform silver nanowires: a plausible growth mechanism and the supporting evidence," Nano Letters, vol. 3, no. 7, pp. 955960, 2003.

[28] X. L. Tang, M. Tsuji, M. Nishio, and P. Jiang, "Roles of chloride anions in the shape evolution of anisotropic silver nanostructures in poly(vinylpyrrolidone) (PVP)-assisted polyol process," Bulletin of the Chemical Society of Japan, vol. 82, no. 10, pp. 1304-1312, 2009.

[29] Z. L. Wang, T. S. Ahmad, and M. A. El-Sayed, "Steps, ledges and kinks on the surfaces of platinum nanoparticles of different shapes," Surface Science, vol. 380, no. 2-3, pp. 302310, 1997.

[30] P. R. Sajanlal, T. S. Sreeprasad, A. K. Samal, and T. Pradeep, "Anisotropic nanomaterials: structure, growth, assembly, and functions," Nano Reviews, vol. 2, p. 5883, 2011.

[31] E. Braun, Y. Eichen, U. Sivan, and G. Ben-Yoseph, "DNAtemplated assembly and electrode attachment of a conducting silver wire," Nature, vol. 391, no. 6669, pp. 775-778, 1998.

[32] K. E. Korte, S. E. Skrabalak, and Y. Xia, "Rapid synthesis of silver nanowires through a $\mathrm{CuCl}$ - or $\mathrm{CuCl}_{2}$-mediated polyol process," Journal of Materials Chemistry, vol. 18, pp. 437-441, 2008.

[33] T. Itakura, K. Torigoe, and K. Esumi, "Preparation and characterization of ultrafine metal particles in ethanol by UV irradiation using a photoinitiator," Langmuir, vol. 11, no. 10, pp. 4129-4134, 1995.

[34] M. A. El-Sayed, "Some Interesting properties of metals confined in time and nanometer space of different shapes," Accounts of Chemical Research, vol. 34, no. 4, pp. 257-264, 2001.
[35] S. Link, M. B. Mohamed, and M. A. El-Sayed, "Simulation of the optical absorption spectra of gold nanorods as a function of their aspect ratio and the effect of the medium dielectric constant," The Journal of Physical Chemistry B, vol. 103, no. 16, pp. 3073-3077, 1999. 

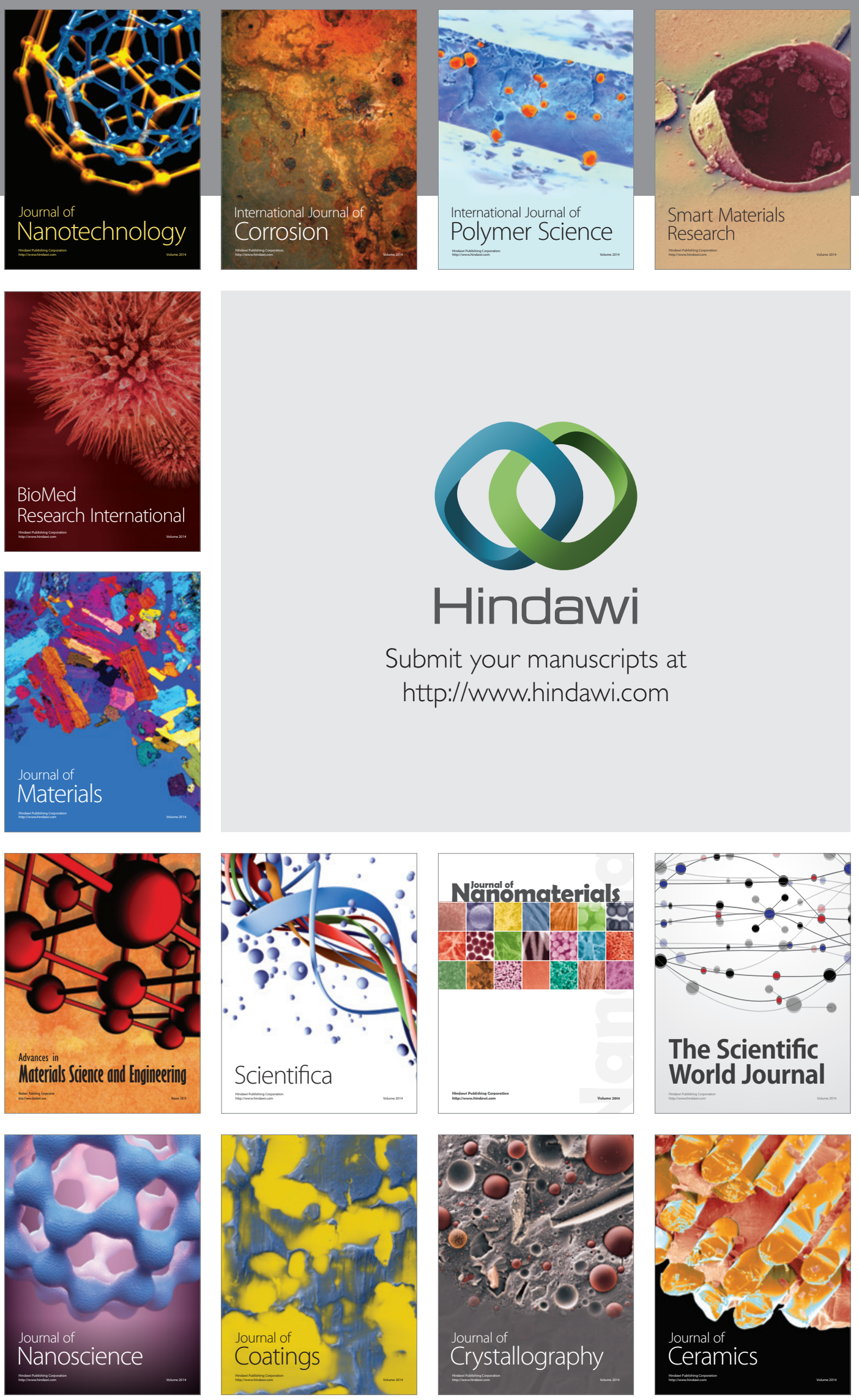

The Scientific World Journal

Submit your manuscripts at

http://www.hindawi.com

\section{World Journal}

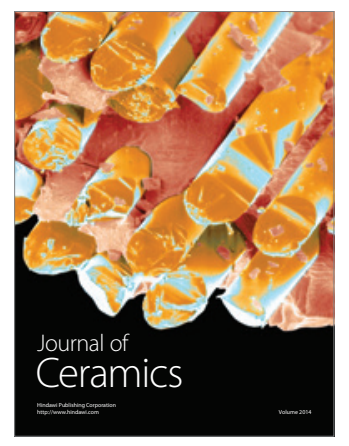

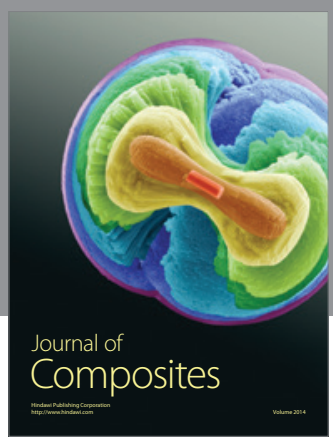
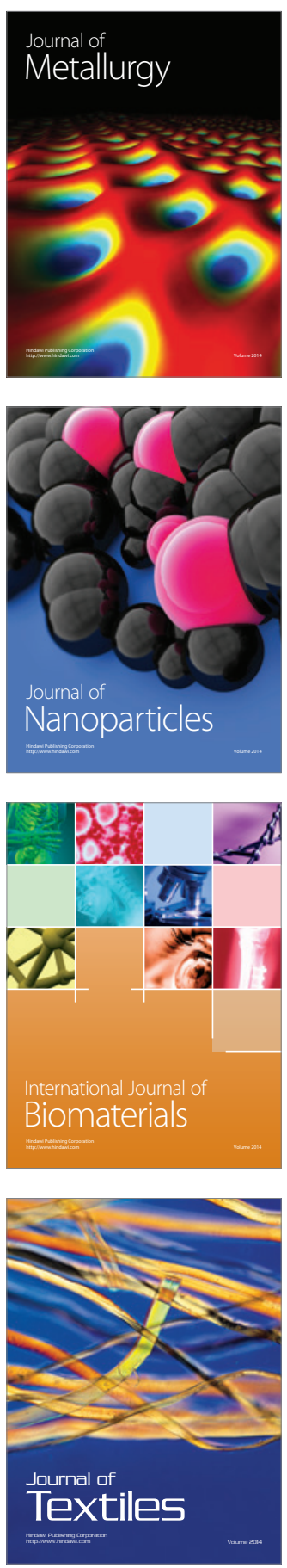